\title{
Dionysisme et démonisme : l'excès dans Macbeth
}

Dionysianism and daemonism in Macbeth

\section{Sélima Lejri}

\section{(2) OpenEdition \\ Journals}

\section{Édition électronique}

URL : http://journals.openedition.org/shakespeare/1066

DOI : 10.4000/shakespeare.1066

ISSN : 2271-6424

Éditeur

Société Française Shakespeare

Édition imprimée

Date de publication : 1 novembre 2007

Pagination : 119-131

ISBN : 2-9521475-4-X

Référence électronique

Sélima Lejri, "Dionysisme et démonisme : l'excès dans Macbeth ", Actes des congrès de la Société française Shakespeare [En ligne], 25 | 2007, mis en ligne le 10 février 2008, consulté le 19 avril 2019. URL : http://journals.openedition.org/shakespeare/1066 ; DOI : 10.4000/shakespeare.1066 


\section{Shakespeare et l'excès}

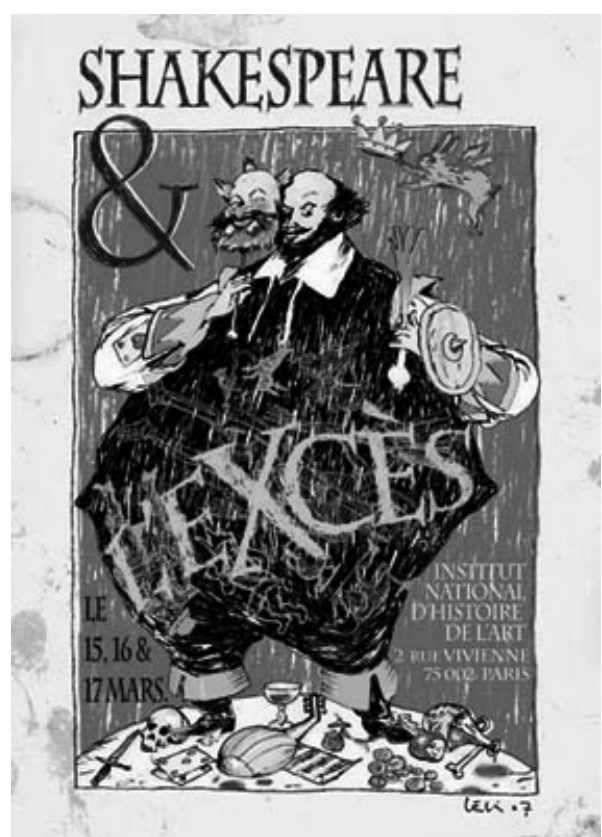

actes du Congrès

organisé par la

SOCIÉTÉ FRANÇAISE SHAKESPEARE

les 15,16 et 17 mars 2007

textes réunis par

Pierre KAPITANIAK

sous la direction de

Jean-Michel DÉPRATS 
COUVERTURE :

Edouard Lekston, Falstaff, 2007

conception graphique et logo

Pierre Kapitaniak

\section{(C) 2007 Société Française Shakespeare \\ Institut du Monde Anglophone}

Université de Paris III - Sorbonne Nouvelle

5 rue de l'École de Médecine 75006 Paris

www.societefrancaiseshakespeare.org

Tous droits de traduction, de reproduction et d'adaptation réservés pour tous les pays 


\title{
DIONYSISME ET DÉMONISME : L'EXCÈS DANS MACBETH
}

\author{
Sélima LEJRI
}

\begin{abstract}
Par delà la lecture judéo-chrétienne où le Mal s'offre comme motif de l'excès, et plus loin que la filiation sénéquéenne de la cruauté, Macbeth semble reprendre le modèle païen grec du ménadisme ou encore du dionysisme, forme extrême de frénésie collective en l'honneur du dieu grec Dionysos. Pour l'Angleterre aux prises avec un mal politique prolifique dont le Complot des Poudres de novembre 1605, la paranoïa qui mine l'État d'Écosse dans la tragédie de Shakespeare est un sentiment bien familier. La fureur homicide qui mène le roi usurpateur du pouvoir à sa déchéance est un tragique par excès vécu sous des auspices assez proches de la fièvre dionysiaque qui ébranle aussi bien la capitale civique Apollinienne, dans l'histoire de la Grèce ancienne, que la Thèbes de Cadmos dans la tragédie d'Euripide, à savoir Les Bacchantes. L'irrépressible phénomène du dionysisme se matérialise dans la représentation mythique du sparagmos, rituel symbolique qui dit la déchirure mentale et psycho-somatique de la victime de Dionysos. Étape incontournable de l'extase ménadique, le dépècement à mains nues de la bête sacrificielle s'érige comme icône trans-culturel de l'essence tragique (donc par delà son contexte proprement grec) et comme force psychopathologique de destruction qui permettent de relier le théâtre shakespearien, en l'occurrence Macbeth, à l'origine intrinsèquement dionysiaque du théâtre.
\end{abstract}

Dionysianism and daemonism in Macbeth Beyond the Judeo-Christian reading according to which Evil figures as the motif of excess, and further back than the Senecan filiation of cruelty, Macbeth seems to revive the pagan motif of maenadism or again dionysianism, an extreme form of collective frenzy in honour of the Greek god Dionysos. Seen from England, which was then grappling with overwhelming political evils culminating in the Gunpowder Plot of November the $5^{\text {th }} 1605$, the paranoia that undermines the Scottish state in the Shakespearian tragedy appeared quite familiar. The homicidal fury that leads the usurping king to his downfall is a tragic experience in a manner quite reminiscent of the Dionysian frenzy which convulses not only the Apollonian civic capital in the history of ancient Greece, but also the Thebes of Cadmos in the tragedy of Euripides, namely The Bacchae. The irrepressible phenomenon of dionysianism operates in the mythical representation of the sparagmos, a symbolic ritual that depicts the mental and psycho-somatic mutilation of the Dionysian victim. Being an inevitable stage of maenadic trance, the bare-arm dismembering of the sacrificial animal emerges as a trans-cultural icon of the soul of tragedy (reaching therefore beyond its properly Greek context) and as a psychopathological drive that help trace Shakespearian drama, namely Macbeth, to the intrinsically Dionysian origin of drama.

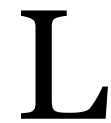

a Renaissance témoigne d'une résurgence allégorique du dieu grec Dionysos sous ses deux facettes: le dieu sage que récupèrent les néoplatoniciens et humanistes comme Marcile Ficin et Pic de la Mirandole pour leur théologie mystique ${ }^{1}$, et le dieu sauvage à la face grimaçante, associé à l'excès et au démonisme comme

\footnotetext{
1 «Aux philosophes que nous serons, Bacchus, le guide des Muses, montrera alors dans ses mystères, c'est-à-dire dans les signes visibles de la nature, les signes invisibles de Dieu... », Pic de la Mirandole, De la Dignité de l'homme, trad. Yves Hersant, Combas, Édition de l'Éclat, p. 33-35.
} 
le Bacchus de Michel-Ange. L'assimilation du dieu à la figure du diable et la comparaison de ses orgies aux sabbats sont des faits attestés par un bon nombre d'historiens ${ }^{2}$. C'est cet aspect là qui fera l'objet de cette étude.

Au-delà de ce retour perceptible du phénomène du dionysisme à la Renaissance, il est possible de comprendre l'action tragique au théâtre comme intrinsèquement dionysiaque de par le lien étroit voire ombilical qui existe entre le genre dramatique et le dieu grec. Dionysos donne leurs tons aux drames satyriques et aux tragédies grecques représentées lors des festivités culturelles connues sous le nom des Grandes Dionysies. Ces tragédies où les extrêmes sont permis mettent en scène des héros en proie à des tourments intérieurs et visualisés par la désarticulation ou la mutilation du corps, souvent sous l'emprise de la démence. Ces personnages semblent revivre ce que les ménades mythiques, accompagnatrices de Dionysos, auraient vécu dans les montagnes: la possession collective au cours de laquelle s'allient chants orgiaques, danse échevelée, chasse et ingestion d'animaux ${ }^{3}$. Le renouveau du dionysisme sous les auspices du démonisme à la Renaissance ainsi que l'essence bachique de la tragédie permettent de comprendre l'excès dans le Macbeth de Shakespeare selon un mode syncrétique où le Mal judéo-chrétien s'aligne sur le modèle païen du dionysisme.

Connu essentiellement comme le dieu du théâtre, du vin et de l'extase (extase double car mystique ou meurtrière selon qu'on est adepte ou pas de ses rites), Dionysos est aussi un dieu à la personnalité insaisissable et qui échappe à toute définition figée en vertu des multiples contradictions qui le caractérisent. Il est l'étranger qui devient familier au cœur de toutes les villes qu'il traverse lors de son périple en Asie et en Orient, notamment à Thèbes comme Euripide le met en scène dans Les Bacchantes ${ }^{4}$. Il est par excellence le dieu qui surgit de nulle part, de l'ailleurs, pour venir balayer de sa fureur la civilité des villes grecques. Son épiphanie est aussi surprenante que

\footnotetext{
2 Notamment par Stuart Clark dans Thinking with Demons : The Idea of Witchcraft in Early Modern Europe, Oxford, Oxford University Press, 1999, p. 23, 333.

3 Voir Diodore de Sicile, Bibliothèque historique, Livre III, Paris, Les Belles Lettres, 1989, LXII.1-10 ; Henri Jeanmaire, Dionysos. Histoire du culte de Bacchus, Paris, Payot, 1970, p. 257-6o.

4 Pour une étude complète des différentes facettes de Dionysos, voir Nathalie Mahé, $L e$ Mythe de Bacchus, Paris, Fayard, 1992.
} 
désastreuse. En dépit de toute surveillance et de tout contrôle, Dionysos, dans la société gréco-romaine aussi bien que dans la tragédie d'Euripide, réussit à implanter son culte, à le propager et, dans le cas des Bacchantes, à détruire la polis ou la cité qui lui résiste. Dans la Grèce fragilisée par la guerre du Péloponnèse (431-404 av. J-C), comme plus tard dans Rome pendant la deuxième guerre punique (219-210 av. J-C), le dionysisme vient recruter des néophytes dans les cités et même dans Athènes, la plus apollinienne d'entre elles. Malgré sa domestication et son intégration surveillée dans le cadre festif des Grandes Dionysies, son débordement demeure inévitable et le nombre des confréries dionysiaques se démultiplie. Tite-Live rapporte dans son Histoire romaine que malgré la loi romaine de 186 av. J-C qui interdit les bacchanales illicites, ces pratiques orgiaques nocturnes continuent de plus belle. L'irruption de Dionysos aussi bien dans le mythe que dans l'histoire rappelle à bien des égards celle des sorcières dans Macbeth. Dans un monde apparemment rationnel, organisé selon des lois pré-établies (notamment un système politique féodal) ou encore régi selon le principe apollinien dans la terminologie nietzschéenne, les sorcières marquent symboliquement l'irruption de l'irrationnel, du chaotique ou encore du dionysiaque ou du dionysien (que Nietzsche oppose au principe apollinien dans La Naissance de la Tragédie) ${ }^{5}$. Mais elles s'introduisent aussi à cause des fissures d'un État fragilisé par la guerre contre l'ennemi norvégien.

De par leur physique androgyne et monstrueux qui les place aux frontières de l'humain, les trois sœurs fatidiques ou «Weird Sisters » offrent une vision dérangeante et transgressive que leurs mots à double-entendre viennent confirmer dans le surnaturel. Elles annoncent un algorithme insolite qui annule toute différence et tout ordre : "fair is foul and foul is fair »(I.i.12). Ce postulat déclenche dans le monde apollinien une logique de l'équivoque que Macbeth adopte dans sa langue énigmatique: "So foul and fair a day I have not seen » (I.iii.36) et exhorte Lady Macbeth à inverser l'ordre

\footnotetext{
5 « Nous aurons fait en esthétique un progrès décisif, quand nous aurons compris, non comme une vue de la raison mais avec l'immédiate certitude de l'intuition, que l'évolution de l'art est liée au dualisme de l'apollinisme et du dionysisme, comme la génération est liée à la dualité des sexes, à leur lutte continuelle, coupée d'accords provisoires », F. Nietzsche, La Naissance de la Tragédie, Traduction française de Michel Haar, Philippe LacoueLabarthe et Jean-Luc Nancy, Paris, Gallimard, 1977, p. 17.
} 
chronologique des choses en lui faisant sentir «the future in the instant » (I.v.56).

Cette perte des différences est de mauvaise augure et annonce ce que René Girard appelle «l'indifférenciation violente » : la chose et son contraire sont attestés, allant au-delà de la confusion linguistique pour préfigurer un décloisonnement dangereux à tous les niveaux: politique, où les rôles roi-vassal sont inversés ; familial, où le rapport père-enfant devient conflictuel (surtout si l'on lit le meurtre de Duncan, figure patriarcale par excellente, comme non seulement régicide mais aussi parricide); sexuel, où les rôles homme-femme sont pervertis (c'est le cas d'un Macbeth pusillanime et féminisé et d'une Lady Macbeth brutale et masculinisée); et enfin, ontologique, où l'humain et l'animal se confondent métaphoriquement (comme le monstre de Banquo ou Macbeth l'ours attaché au poteau) ${ }^{6}$.

Cet effacement des différences, c'est le propre de Dionysos et de son culte. Il est celui qui fait tomber toutes les barrières, entre les sexes, entre les jeunes et les vieux, entre le dieu, l'homme et l'animal, afin d'inoculer le germe d'une folie généralisée. La violence qu'il sème est contaminatrice et n'épargne personne ${ }^{7}$. Par ailleurs, l'apparition des sorcières dans Macbeth marque la manifestation du mystère ou de l'étrange que suggère déjà l'épithète "weird ». C'est leur étrange information - «strange intelligence» (I.iii.74) - concernant la consécration de Macbeth seigneur de Cawdor qui fait s'infiltrer l'étrange ou l'étrangeté par les fissures d'un royaume fragilisé par la guerre avec l'ennemi norvégien. Les exploits guerriers de Macbeth sont qualifiés de "strange images of death" (I.iii.95) et le meurtre de Duncan, commis par une nuit des plus étranges dont témoignent Ross et un vieil homme (II.iv.1-4), est rendu par une image insolite où le corps gisant du vieux roi est comparé à la Gorgone, figure d'effroi où se concentrent l'humain et le bestial et dont le regard meurtrier fait basculer son contemplateur dans l'Hadès souterrain. Frappées de surnaturel, et porteuses d'étrangeté, les sorcières ainsi que leur avatar la Gorgone rappellent à bien des égards la figure du dieu grec Dionysos

${ }^{6}$ Voir William Shakespeare, Macbeth, éd. A. R. Braumnmuller, coll. The New Cambridge Shakespeare, Cambridge, Cambridge University press, 1997, III.iv.100-103 ; v.vii.1-2.

7 Voir Euripide, Les Bacchantes dans Tragédies Complètes II, éd. Marie-Delcourt-Curvers, Paris, Gallimard, coll. Folio Classique, 1962, v. 216-241 ; René Girard, La Violence et le Sacré, Paris, Grasset, coll. Hachette Littératures, 1972, p. 181-84. 
que Marcel Detienne qualifie de «l'étrange porteur d'étrangeté ${ }^{8}$ ». Il incarnerait le unheimlich ou « l’inquiétante étrangeté » freudienne, ce familier qui, longtemps inhibé dans l'inconscient, remonte à la surface et apparaît dès lors comme étrange et effrayant ${ }^{9}$. Sans pour autant le pousser au crime, les sorcières déclenchent en quelque sorte la part dionysiaque en Macbeth et font surgir ce familier qui l'habite mais que l'oubli a rendu étrange et étranger. La prédiction qu'il sera roi d'Écosse fait surgir à la surface de sa mémoire le souvenir de choses longtemps oubliées: "My dull brain was wrought / With things forgotten » (I.iii.148-149). Macbeth donne un nom à cette force irrationnelle et étrange qui ravit son esprit (Banquo et lui-même plus tard utilisent le mot « rapt » I.iii.141, I.v.5). Cette force c'est son ambition qu'il dit être viscérale et débordante. Il en donne une image équestre bien particulière :

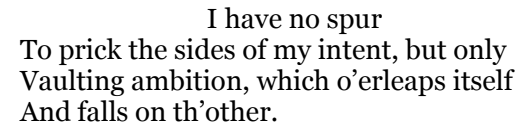

(I.vii.25-28)

Dans cette métaphore, l'ambition est le cavalier pressé qui, pour vouloir être trop vite en selle, retombe de l'autre côté de son cheval. Cette ambition a des relents démoniaques car le cheval est traditionnellement associé au monde chtonien et au démon qui l'aurait choisi comme monture. Dans la légende écossaise, plus précisément, le kelpie est un démon aquatique à la forme hippique et qui hante le Loch Ness. Par ailleurs, Macbeth rappelle à bien des égards Phaéton, figure de l'insouciance juvénile dans la mythologie grecque qui s'empare du char de son père Hélios et qui, ne maîtrisant pas les rênes, embrase la terre.

C'est en donnant libre cours à cette ambition effrénée que Macbeth s'abîme dans la fureur homicide. Il devient le mauvais démiurge de lui-même, son propre esprit possesseur, ou encore son propre démon dans le sens grec du terme, daîmon, le dieu malin ou le surnaturel destructeur, la face maléfique du theos ${ }^{10}$. Son «auto-

\footnotetext{
${ }^{8}$ Dionysos à ciel ouvert, Paris, Hachette, 1986, p. 33.

${ }^{9}$ Voir Sigmund Freud, L'Inquiétante étrangeté et autres textes, trad. Fernand Cambon, Paris, Gallimard, 2001, p. 33-37, 99.

${ }^{10}$ Sur le concept du daîmon ou dæmon depuis Homère voir Edmond M. Saglio et C. Daremberg, Dictionnaire des antiquités grecques et romaines, Paris, Hachette, 1892.
} 
possession » diabolique peut donne lieu à ce qu'il nomme « my strange and self-abuse » (III.iv.143). Le « self-abuse » fait certes référence au spectre de Banquo que Macbeth chasse comme une simple hallucination mais a aussi des relents diaboliques car « abuse » (verbe et nom) est la prédilection du Diable contre lequel Jacques vI d'Écosse ne cesse de mettre en garde dans son traité de démonologie ${ }^{11}$. Cet esprit possesseur à la dimension humaine germe en Macbeth, grandit en lui mais aussi le dépasse, le transcende, au point de devenir une force surnaturelle qui agit fatalement sur lui. L'excès dans lequel Macbeth sombre est paradoxalement hybris fatale, démesure et arrogance humaines qui s'articulent comme une force incoercible et hypnotique et qui le pousse inexorablement vers sa perte, avec et contre son gré.

$\mathrm{Au}$ moment où il cède à cette pulsion qu'il nomme «vaulting ambition » (I.vii.27), il se laisse envahir par ses propres « daemonic / Dionysian energies", analogie que fait Marvin Rosenberg à son propos $^{12}$. Toutefois, c'est sous l'égide de son épouse que ces énergies sont exacerbées. Le croisement entre son hybris personnelle et celui de sa femme donne naissance aux deux monstres ou diables: Macbeth «this fiend of Scotland (Iv.iii.236) et Lady Macbeth « his fiend-like queen » v.ix.36).

Lady Macbeth vit symboliquement l'excès sous le mode de la privation et de la mutilation. Lorsqu'elle invoque les esprits maléfiques, elle espère voir s'effacer en elle tout trait féminin et maternel et se projette dans un être stérile et asexué. Aux flux des menstrues et du lait elle préfère l'endurcissement du sang coagulé et l'acidité du fiel : «make thick my blood » et «take my milk for gall ». Elle prie pour l'obstruction de tout son être et son engorgement de la pire cruauté de peur que la pitié ou le remords ne l'atteigne :

\footnotetext{
11 « But may it not suffice him to have indirectly the rule, and procure the perdition of so manie soules by alluring them to vices, and to the following of their own appetities, suppose he abuse not so many simple soules, in making them directlie acknowledge him for their maister », liv. I, chap. 2 / " [...] all Devils must be lyars; but so they abuse the simplicitie of these wretches, that becomes their schollers, that they make them beleeve, that at the fall of Lucifer, some Spirites fell in the air, some in the fire, some in the water, some in the lande: In which Elementes they still remaine ", liv. I, chap. 6. Jacques vi d'Écosse, Dæmonologie dans Minor Prose Works of King James VI and I, éd. James Craigie, Édimbourg, Scottish Text Society, 1982 (1 $1^{\text {re }}$ éd. 1597).

12 Marvin Rosenberg, Masks of Macbeth, Los Angeles et Londres, University of California Press, 1978, p. 95 et p. 74 .
} 
Stop up th'access and passage to remorse
That no compunctious visitings of nature
Shake my fell purpose nor keep peace between
Th'effect and it.
(I.v.42-45)

Elle ressort de ce rite de passage métamorphosée en monstre dénaturé et diabolisé. Par ailleurs, elle se projette dans la marâtre infanticide capable de tuer l'enfant qui tète son sein, rappelant ainsi la figure de la ménade possédée de Dionysos qui lacère de ses propres mains le corps de son enfant, comme les filles de Minyas dans une des légendes grecques ou Agavé dans Les Bacchantes d'Euripide. Ainsi transformée, elle réussit à pervertir la nature de son mari et à lui faire dépasser les limites de sa virilité, mais surtout de son humanité :

When you durst do it, then you were a man.

And to be more than what you were, you would

Be so much more the man.

À force de prendre pour modèle sa maîtresse, le disciple Macbeth le dépasse. Au bout de son parcours criminel, il s'érige lui-même en monstre impassible, aussi dénaturé et déshumanisé que son épouse. Le trop-plein de tourments lancinants dont il donne l'image expressive : « $\mathrm{O}$, full of scorpions is my mind, dear wife! » (III.ii.36) ; cède à l'indifférence inhumaine, protection acquise grâce au surplus de l'horreur qu'il finit par accumuler :

I have supped full with horrors

Direness familiar to my slaughterous thoughts

Cannot once start me.

(V.v.13-15)

S’il a des motifs logiques pour tuer ses premières victimes (Duncan dont il convoite la couronne et Banquo, témoin et rival des prophéties des sorcières), Macbeth n'a aucune raison "plausible » pour s'attaquer à la famille de Macduff. Toutefois, dans la folie homicide qui le mine, il s'abîme dans le meurtre avec la frénésie d'un dément que rien ne peut plus arrêter. Le seul fait que Macduff n'assiste pas à la fête de son intronisation (III.iv.128-29) sème en lui une peur paranoïaque vite supplantée par la conviction rationalisée que celui-là est un ennemi qu'il lui faut traquer sans tarder. Incapable de mettre la main sur Macduff, il ordonne la mise à mort de l'épouse et des enfants de celui-ci. Le crime devient pour lui une action préventive, une nécessité fastidieuse et une impulsion morbide. Empêtré dans le sang 
de ses victimes, Macbeth ne choisit plus sa voie, mais s'abandonne au courant qui l'entraîne vers sa perte :

I am in blood

Stepped in so far that should I wade no more,

Returning were as tedious as go o'er.

(III.iv.136-38)

Il s'enhardit alors dans une logique du pire, ou encore, dans un tragique par l'excès à l'opposition d'un Hamlet qui s'aveulit dans un tragique par défaut (j'emprunte ces deux formules à Christine BuciGlucksmann) ${ }^{13}$. Le crime finit par être une idée fixe ou une monomanie qui obsède l'esprit de Macbeth et qui loin d'être réfléchi devient compulsif : "Strange things I have in head that will to hand / Which must be acted ere they may be scanned » (III.iv.139-40).

$\mathrm{Au}$ comble de sa fureur meurtrière, Macbeth souhaite que toute vie autour de lui soit tuée : «Whiles I see lives, the gashes / Do better upon them " (v.viii.2-3). Son excès tragique ne se dessine pas en crescendo linéaire mais en boucle infernale où folie et crime alternent et deviennent si intimement liés qu'ils finissent par se confondre. Cet excès semble opérer sur un mode dionysiaque où folie et (mania et sparagmos) forme le dénominateur commun de presque toutes les légendes rattachées au dionysisme : le dieu inflige la folie à sa victime, généralement pour avoir négligé ou méprisé ses rites. En proie à la démence, celle-ci commet un meurtre. Pour avoir perpétré ce meurtre, cette dernière est encore frappée de folie. Le meurtre fait donc office d'une charnière entre folie punitive et folie expiatrice. Dans le cas des Bacchantes d'Euripide, Penthée et sa mère Agavé subissent la folie punitive de Dionysos pour s'être refusés à sa religion. Penthée cherche à épier les rites auxquels il n'est pas initié et, en guise de récompense, le dieu lui inocule la fièvre dionysiaque, l'envoie sur le Cithéron et le jette en pâture à sa mère et aux thébaines en furie qui écartèlent son corps. Les deux sont ainsi châtiés : le fils est victime de la mère et réciproquement la mère est victime de l'infanticide qu'elle commet et qui la plonge dans une dépression démentielle encore plus grande.

L'humeur dans laquelle Macbeth sombre a des accents étrangement dionysiaques. Le héros connaît les affres de l'extase au sens étymologique du terme, ex-stasis, l'état hors de soi ou précisément la raison délogée : il parle de « restless ecstasy » (III.ii.22).

13 Tragique de l'ombre. Shakespeare et le maniérisme, Paris, éd. Galilée, 1990, p. 85. 
Cette dépossession de soi supplantée par l'envahissement du démoniaque semble être une réactualisation de l'expression dramatique primitive, celle que lègue le Dionysos mythique à la scène grecque du v siècle av. J-C. Cette expression est celle de la division, de la dépersonnalisation et de la déchirure mentale. Elle trouve son pendant iconique ou sa métonymie visuelle dans le rituel dionysiaque du sparagmos, la dilacération d'un animal, voire d'une victime humaine, à mains nues. Outre son signifié relatif à la religion dionysiaque, ce rituel acquiert le sens métaphorique de scission ou d'éclatement mental, ou ce que la psychiatrie nommera plus tard schizophrénie. Dionysos, auteur de ce rituel, devient lui-même la figure emblématique de cet état mental. Vidé de ses attributs mythiques, il s'annonce comme la force destructrice de tout héros tragique, voire le concept psychopathologique de la raison fragmentée ${ }^{14}$. La forme extrême de la transe dionysiaque est celle d'une mort cruelle et crue qu'on subit ou qu'on inflige. C'est par le truchement du corps que l'excès du délire est exprimé dans sa littéralité. Séparés d'eux-mêmes et dépossédés de leur raison, Macbeth et son épouse encourent la forme extrême de la déchirure psychosomatique: elle se suicide, probablement par pendaison, il tombe sous le coup de grâce porté par Macduff. Les deux corps subissent l'ultime déchirure aux relents dionysiaques: tous les excès confondus sont ici transcrits dans la décapitation de Macbeth par Macduff et dans ce geste violent que Lady Macbeth s'inflige («by self and violent hands / took off her life», v.ix.37-38).

La mise à mort de Macbeth marque la fin d'un interlude politique chaotique. Il est néanmoins possible de se demander si cette action, ponctuée par le couronnement de Malcolm qui symbolise le renouveau royal, met réellement fin à toute violence potentielle future. Le germe de l'infection (pour reprendre les mots du docteur qui parle de «infected minds", v.i.62) peut s'être répandu aux alentours. Malcolm ne cacherait-il pas un autre Macbeth ? Le futur roi d'Écosse

\footnotetext{
14 «the signature mark of the god's [Dionysus'] impress - the rending of character and the literal or metaphorical sparagmos - can be identified in any age when tragic dramas have been written. Each of these terms - the daemonic and the Dionysian - can be understood as denoting a tragic field [...] each one is productive of the distinctly tragic effect of interior rending and disjuncture. » William Storm, After Dionysus. A Theory of the Tragic, Ithaca, Cornell University Press, 1998, p. 115-16.
} 
dit qu'il a quelque chose de Macbeth en lui : "I am young, but something / You may discern of him through me... », (Iv.iii.14-15).

S'il l'on considère les propos de Malcolm pendant le test qu'il fait subir à Macduff pour sonder sa loyauté (Iv.i), on demeure sceptique quant à son honnêteté même s'il révèle, par la suite, que toutes les fautes dont il s'est affublé ne sont que purs mensonges. Dans cette fable qu'il tisse à son propos, il dépeint le portrait d'un être ignoble dont les vices dépasseraient ceux de Macbeth au point que «black Macbeth / Will seem as pure as snow » (Iv.iii.52-53). Contre les abus excessifs de Macbeth, Malcolm offre des maux sans limites (« confineless harms », 55) et annonce une concupiscence sans bornes (« But there's no bottom, none, / in my voluptuousness », 60-61). Tel le tonneau des Danaïdes, la citerne de ses désirs ( «the citern of my lust »,63) ne pourrait jamais se remplir et le rassasier. Tous les excès du tyran seraient outrepassés par Malcolm. Le torrent de violence que connaît l'Écosse n'en serait que fortifié sous son règne :

When I shall tread upon the tyrant's head,

Or wear it on my sword, yet my poor country

Shall have more vices than it had before,

More suffer, and more sundry ways than ever,

By him that shall succeed.

(IV.iii.45-49)

Macduff a beau repousser les limites de l'acceptable et du compréhensible en promettant des plaisirs à foison qui pourraient satisfaire et combler le futur roi, ce dernier s'obstine à renchérir sur le transgressif et à décupler le déjà excessif avant de ponctuer cette noire allégorie de la pire note macabre :

\footnotetext{
Nay, had I power, I should

Pour the sweet milk of concord into hell,

Uproar the universal peace, confound

All unity on earth.
}

(IV.iii.97-100)

Ce chaos cosmique que projette Malcolm sous son règne peut être lu à la lumière de la "mimesis violente ", mécanisme reconnu par René Girard comme découlant de la symétrie, de la réciprocité et des représailles qui animent l'affrontement des rivaux ${ }^{15}$. Toutefois, loin d'être une réponse par l'identique, par le même envers Macbeth, le

15 René Girard, La Violence et le Sacré, p. 75. 
jeune prince offre une imitation par l'excès, un miroir grossissant, un mal qui renchérit sur celui de Macbeth.

Face à ces promesses alarmantes, Macduff manifeste le plus grand désarroi et déplore le sort funeste de sa patrie. Malcolm finit alors par avouer que ces tares ne sont que fictions. Perplexe devant ce revirement si soudain, Macduff fait planer le mystère sur la personne habilement versatile de Malcolm: "Such welcome and unwelcome things at once, / 'Tis hard to reconcile » (Iv.iii.138-39).

Cet épisode mystérieux où Malcolm se diabolise puis s'innocente avec beaucoup d'aisance laisse en suspend la réponse quant à la nature de son futur règne. Il peut être le règne de l'anarchie et de l'excès comme celui de la sagesse mesurée de la tempérance ou sophrosune apollinienne qu'il revendique («modest wisdom», Iv.iii.119)

L’inquiétude de Macduff laisse ainsi supposer que le cycle de la traîtrise pourrait de nouveau être amorcé après l'éradication du mal que tous imaginent concentré uniquement en Macbeth. Si Macdonwald cachait un traître en Macbeth, celui-ci pourrait bien cacher un nouveau traître en Malcolm. Et chacun d'entre eux semble renchérir sur le précédent. Macdonwald n'est pas pire que Macbeth et Macbeth qu'on croit avoir atteint le seuil maximum de la « diablerie » (« Not in the legions / Of horrid hell can come a devil more damned / In evils to top Macbeth », Iv.iii.55-57), est loin d'être pire que Malcolm dans la première version qu'il donne de lui-même.

Ainsi la conclusion formelle de Macduff qui signale que «the time is free » (v.ix.22) rivalise avec le doute que laisse planer la tête tranchée de Macbeth. Il faut l'imaginer plantée sur les remparts aux côtés de celle de Macdonwald, signalant que le corps du traître n'est plus mais que son esprit vit peut être en quelqu'un d'autre et que sa souillure risque de se répandre à nouveau. La violence peut donc affleurer à tout moment et, comme la fièvre de la bacchanale, elle menace de sévir envers et contre toute répression. Ross l'aurait prédit :

I dare not speak much further, But cruel are the times when we are traitors And do not know ourselves, when we hold rumour

From what we fear, yet know not what we fear, But float upon a wild and violent sea,

Each way and none. 
Ross dépeint une violence endémique et une paranoïa contaminatrice où tout un chacun est un traître potentiel. Dans cette réflexion psychanalytique avant l'heure, la traîtrise n'est plus un vice que l'on cache aux autres mais un mal qui existe à l'insu du traître même, et la peur n'a plus d'objet mais devient une finalité. Elle n'est donc plus canalisée vers un ennemi précis, mais projetée dans tous les sens, vers une multitude de dangers virtuels, aussi substantiels que le fantôme de Banquo.

Dans l'Angleterre récemment secoué par le Complot des Poudres de novembre 1605, les appréhensions de Ross traduisent la mentalité d'un peuple aguerri. La vague de violence exprimée par " wild and violent sea" agit comme la fièvre de la bacchanale qui déferle sur les villes grecques, dans l'histoire, le mythe et la littérature (du moins, dans la tragédie d'Euripide), fièvre qui se fortifie là-même où elle est réprimée. Comme il est signalé plus haut, la répression des Bacchanales et les tentatives d'épuration du phénomène dionysiaque par le sénat de Rome au $\mathrm{II}^{\mathrm{e}}$ siècle av. J-C s'avérèrent vaines. L'image assagie de Bacchus/Liber Pater que les autorités essayèrent de cultiver (en mettant en avant ses qualités agraires) ne parvinrent pas à occulter ou supplanter celle de Dionysos, le dieu de l'extase et de tout débordement parfois dangereux.

Après avoir ainsi essayé de dégager les différentes articulations de l'excès dans Macbeth, il serait possible de réfléchir quant à la démarcation de cette œuvre qui semble outrepasser l'idée même de la Tragédie (au sens Aristotélicien du terme). Ce qui la place au-delà du tragique ce n'est pas seulement la ramification de la violence et de la peur, mais surtout l'annulation de leur causalité (Macbeth est violent et Macbeth a peur et dans la réflexion de Ross, tout le monde est violent et tout le monde a peur). Dans Les Bacchantes d'Euripide, la cruauté et la souffrance sont réparties entre le dieu Dionysos et ses victimes, Agavé et Penthée. Dans la pièce de Shakespeare, la bipolarité de l'horreur, représentée par la cruauté et la souffrance, est vécu par le héros qui est victime de son démiurge, de son propre Dionysos. La transcendance de soi dans la possession démoniaque (et qui implique la cruauté) et la désintégration de soi par cette même possession désintégration jusque dans la chaire mutilée, signalant la souffrance porte l'esthétique de Macbeth au-delà du tragique, dans une catégorie à laquelle Ekbert Faas donne le nom de «post-tragic », où la cruauté et 
la souffrance ne sont ni significatives, comme dans la tragédie, ni gratuites, comme dans l'anti-tragédie, mais où elles sont, en soi et pour soi ${ }^{16}$. Le tragique en excès des Bacchantes d'Euripide se trouve ici excédé.

Sélima LEJRI

Université de Tunis

${ }^{16}$ Ekbert Faas, Tragedy and After : Euripides, Shakespeare and Goethe, Kingston, Montreal, McGill-Queen's University Press, 1984, p. 4-24. 